\title{
Indicativos de algumas alometrias do peixe Deuterodon hastatus (Characidae)
}

\author{
June Cunha de Araujo (D
}

\author{
Rosa García Márquez(D) \\ Rosana Souza-Lima (D)
}

\author{
Jorge Corrêa de Araújo (D)
}

\section{Resumo}

A alometria é o estudo das relações de padrões de crescimento entre indivíduos de uma mesma espécie, que são estabelecidas por meio da análise da proporcionalidade que envolve o crescimento específico de determinados órgãos ou partes do corpo de indivíduos de uma determinada espécie. Neste trabalho certos padrões de crescimento entre órgãos de peixes da espécie Deuterodon hastatus (Characidae) foram observados empiricamente e confirmados, posteriormente, como um indicativo da lei de alometria. Alguns exemplares dessa espécie de peixe foram coletados na bacia do rio Aldeia que corre entre os municípios de São Gonçalo e Itaboraí, localizados no leste da região metropolitana do estado do Rio de Janeiro. As taxas de crescimento envolvendo a proporcionalidade entre os órgãos observados foram estimadas por meio de uma regressão linear para cada caso analisado. Nesse estudo, um indicativo de alometria foi obtido por meio de três metodologias distintas, sendo uma delas denominada como projeção ortogonal, a qual permite obter de modo mais fácil os valores calculados a partir do modelo de alometria estabelecido, determinando assim a reta de ajuste com os dados experimentais. Os valores calculados a partir do modelo estabelecido para a comparação entre o comprimento e a altura do corpo do peixe - mostraram boa concordância com os valores observados.

Palavras-chave: Proporções corporais; Método dos mínimos quadrados; Regra de Cramer; Coeficiente de determinação; Actinopterygii.

\section{Abstract}

Allometry is the study of growth pattern relationships between individuals of the same species that are established through the analysis of proportionality that involves the specific growth of certain organs or body parts of individuals of a given species. In this work certain growth patterns among fish organs of the species Deuterodon hastatus (Characidae) were observed empirically and confirmed later, as an indication of the law of allometry. Some specimens of this fish species were collected in the Aldeia river basin that runs between the municipalities of São Gonçalo and Itaboraí, located in the east of the metropolitan region of the state of Rio de Janeiro. Growth rates involving the proportionality between the observed organs were estimated using linear regression for each case analyzed. In this study, an indicative of allometry was obtained through three different methodologies, one of which is called orthogonal projection, which makes it easier to obtain the values calculated from the established allometry model and determines the fit line with the data experimental tests. The values calculated from the established model for the comparison between the length and height of the fish's body showed good agreement with the observed values. 
Keywords: Body proportions; Minimum squares method; Cramer's rule; Determination coefficient; Actinopterygii.

\section{Introdução}

A espécie de peixe Deuterodon hastatus (Myers 1928) é comumente encontrada em riachos costeiros do estado do Rio de Janeiro (Figura 1). Essa espécie não tem sido objeto de muitos estudos, e poucos são os dados disponíveis sobre sua ontogenia, história de vida, dinâmica populacional e papel nas cadeias tróficas dos ecossistemas em que vive [9]. Estudos cromossômicos sugerem, ainda, que se trate de um complexo constituído por mais de uma espécie, incluindo formas muito semelhantes entre si [8].

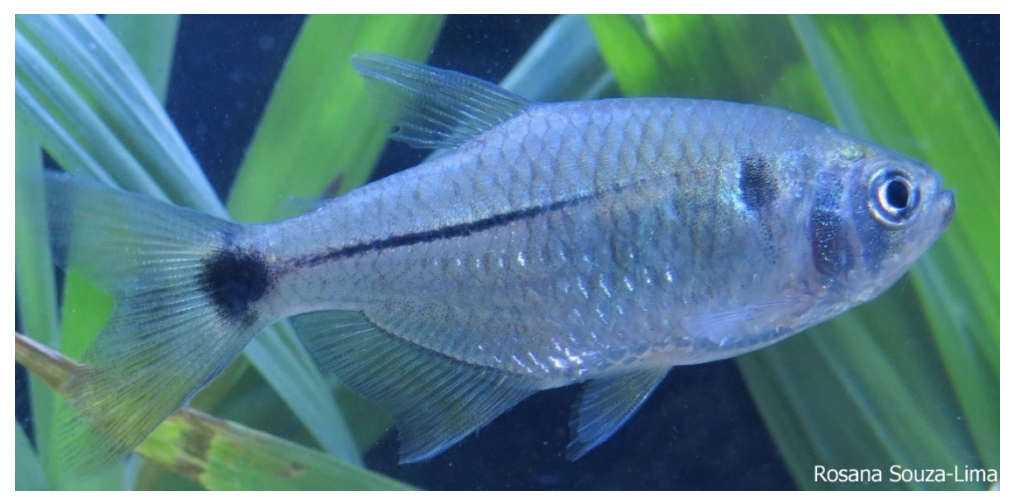

Figura 1: Exemplar de peixe da espécie Deuterodon hastatus (Myers, 1928), com comprimento igual a $55.02 \mathrm{~mm}[12]$, investigada nesse trabalho.

O objetivo desse estudo consiste em obter indicativos de relações alométricas entre machos e fêmeas dessa espécie, com base na regressão linear por meio de diferentes metodologias.

\section{Lei de alometria}

O substantivo "alometria" foi designado por Huxley e Teissier em 1936 [10] visando descrever o estudo dos diferentes padrões de crescimento de uma parte do organismo em relação à outra parte ou ao seu conjunto.

Sejam $\mathrm{x}(\mathrm{t})$ e $\mathrm{y}(\mathrm{t})$ o tamanho de duas partes distintas do organismo de um mesmo indivíduo, em um tempo t. Por tamanho de uma parte ou órgão, podemos considerar o volume, peso, comprimento, área lateral, altura da cabeça, diâmetro do olho, largura da boca, entre outros. De modo a comparar os diferentes crescimentos de órgãos, usaremos o crescimento específico de cada órgão, dado por

$$
\frac{1}{\mathrm{x}} \frac{\mathrm{dx}}{\mathrm{dt}} ; \mathrm{x}>0
$$

onde $\mathrm{x}(\mathrm{t})$ indica o tamanho desse órgão no tempo t.

A Lei da Alometria estabelece que, no mesmo indivíduo, "os crescimentos específicos de seus órgãos 
são proporcionais" [2]. O modelo matemático para a representação dessa lei é então dado por

$$
\frac{1}{\mathrm{y}} \frac{\mathrm{dy}}{\mathrm{dt}}=\mathrm{b} \frac{1}{\mathrm{x}} \frac{\mathrm{dx}}{\mathrm{dt}},
$$

onde $\mathrm{x}>0, \mathrm{y}>0$ e b uma constante denominada de taxa associada ao crescimento relativo [2], também chamada de expoente ou coeficiente alométrico. Pela regra da cadeia, a equação (2) pode ser escrita como

$$
\frac{\mathrm{dy}}{\mathrm{dx}}=\mathrm{b} \frac{\mathrm{y}}{\mathrm{x}}
$$

A equação diferencial linear ordinária (3) pode ser resolvida pelo método de separação de variáveis dada pela integração simples realizada membro a membro dada por

$$
\int \frac{d y}{y}=\int b \frac{d x}{x} .
$$

Da equação (4), resulta

$$
\ln \mathrm{y}=\mathrm{b} \ln \mathrm{x}+\ln \mathrm{a},
$$

onde a é uma constante arbitrária positiva. Aplicando a função exponencial a ambos os membros da equação (5), resulta

$$
\mathrm{y}=\mathrm{ax} \mathrm{x}^{\mathrm{b}}
$$

Os padrões de crescimento representados pelas constantes a e b capturam a relação de proporcionalidade entre dois órgãos de indivíduos de uma mesma espécie. Se na equação (5) fizermos

$$
\mathrm{X}=\ln \mathrm{x} \text { e } \mathrm{Y}=\ln \mathrm{y}
$$

a equação (5) transforma-se em uma equação linear da forma

$$
\mathrm{Y}=\alpha_{1} \mathrm{X}+\alpha_{2}
$$

onde $\alpha_{1}=$ b e $\alpha_{2}=\ln$ a. A equação (8) é a equação de uma reta com inclinação $\alpha_{2}=\mathrm{b}$, chamada de coeficiente alométrico [2].

As relações alométricas ocorrem em diversos níveis de organização biológica, sendo que nesse trabalho vamos nos restringir à avaliação do crescimento relativo de duas partes de indivíduos de uma mesma espécie.

\section{Método de Regressão Linear}

A regressão linear faz parte de uma teoria mais abrangente, denominada método dos mínimos quadrados lineares, que é uma técnica de aproximação muito usada na análise numérica e em problemas práticos como na física, biologia, engenharia, química, entre outras ciências. O método dos mínimos quadrados foi publicado pela primeira vez, por Adrien-Marie Legendre (1752-1833) em 1805, mas já era usado por Carl Friedrich Gauss (1777-1855) [4]. O objetivo da regressão linear é obter correlações ou não entre diferentes variáveis quantitativas. Esse método consiste em buscar aproximações para dados que são obtidos experimentalmente com um certo grau de imprecisão. 
Esse esquema será utilizado nesse estudo para a obtenção dos parâmetros envolvidos na equação (8) do tipo log - log de modo a encontrar os parâmetros de natureza biológica dados pela equação (6).

A regressão linear consiste em encontrar uma reta que melhor se ajuste ao conjunto de pontos $\left(\mathrm{x}_{\mathrm{i}}, \mathrm{y}_{\mathrm{i}}\right), \mathrm{i}=1, \ldots, \mathrm{m}$, coletados ou obtidos de forma teórica ou prática. Por isso, é interessante a obtenção de várias formas ou procedimentos disponíveis para sua determinação. Neste sentido são apresentadas três metodologias distintas com base na minimização do funcional dado pela equação

$$
\mathrm{F}(\alpha)=\mathrm{F}\left(\alpha_{1}, \alpha_{2}\right)=\sum_{\mathrm{i}=1}^{\mathrm{m}}\left[\mathrm{f}\left(\mathrm{x}_{\mathrm{i}}\right)-\varphi\left(\mathrm{x}_{\mathrm{i}}\right)\right]^{2},
$$

onde $\mathrm{f}\left(\mathrm{x}_{\mathrm{i}}\right)=\mathrm{y}_{\mathrm{i}}$ com $\mathrm{i}=1, \ldots, \mathrm{m}$, enquanto $\varphi(\mathrm{x})$ é a função linear de aproximação da forma

$$
\varphi(\mathrm{x})=\alpha_{1} \mathrm{~g}_{1}(\mathrm{x})+\alpha_{2} \mathrm{~g}_{2}(\mathrm{x})
$$

onde $\mathrm{g}_{1}(\mathrm{x})=\mathrm{x} \mathrm{e} \mathrm{g}_{2}(\mathrm{x})=1 ; \mathrm{x} \in[\mathrm{a}, \mathrm{b}] \subset \mathbb{R}$. O objetivo da escolha dessas funções é porque elas formam uma base para o espaço vetorial das funções lineares definidas nesse intervalo.

Denotemos por $\overline{g_{1}}, \overline{g_{2}}$ e $\bar{f}$ os vetores de $\mathbb{R}^{m}$ definidos por

$$
\overline{g_{1}}=\left(g_{1}\left(x_{1}\right), g_{1}\left(x_{2}\right), \ldots, g_{1}\left(x_{m}\right)\right)^{T} ; \overline{g_{2}}=\left(g_{2}\left(x_{1}\right), g_{2}\left(x_{2}\right), \ldots, g_{2}\left(x_{m}\right)\right)^{T}
$$

$\mathrm{e}$

$$
\overline{\mathrm{f}}=\left(\mathrm{f}\left(\mathrm{x}_{1}\right), \mathrm{f}\left(\mathrm{x}_{2}\right), \ldots, \mathrm{f}\left(\mathrm{x}_{\mathrm{m}}\right)\right)^{\mathrm{T}},
$$

onde $\mathrm{x}_{\mathrm{i}} \in[\mathrm{a}, \mathrm{b}] ; \mathrm{i}=1, \ldots, \mathrm{m}$.

A seguir apresentaremos as metodologias para a regressão linear com base na minimização do funcional dado pela equação (9) baseada no método dos mínimos quadrados.

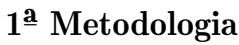

Considere a função de aproximação dada na equação $(10)$, onde $\mathrm{g}_{1}(\mathrm{x})=\mathrm{x}$ e $\mathrm{g}_{2}(\mathrm{x})=1$ com $\varphi(\mathrm{x})$ definida em [a,b]. Então, a equação (9) pode ser escrita da forma

$$
\mathrm{F}\left(\alpha_{1}, \alpha_{2}\right)=\sum_{\mathrm{i}=1}^{\mathrm{m}}\left(\mathrm{y}_{\mathrm{i}}-\alpha_{1} \mathrm{x}_{\mathrm{i}}-\alpha_{2}\right)^{2}
$$

Da equação (13) tem-se as equações dos pontos críticos dadas por

$$
\frac{\partial}{\partial \alpha_{1}} \mathrm{~F}\left(\alpha_{1}, \alpha_{2}\right)=0 \quad \text { e } \quad \frac{\partial}{\partial \alpha_{2}} \mathrm{~F}\left(\alpha_{1}, \alpha_{2}\right)=0 .
$$

As expressões dadas pela equação (14) podem ser colocadas na forma

$$
\frac{\partial}{\partial \alpha_{1}} \mathrm{~F}\left(\alpha_{1}, \alpha_{2}\right)=-2 \sum_{\mathrm{i}=1}^{\mathrm{m}}\left(\mathrm{y}_{\mathrm{i}}-\alpha_{1} \mathrm{x}_{\mathrm{i}}-\alpha_{2}\right) \mathrm{x}_{\mathrm{i}}=0 \quad \text { e } \quad \frac{\partial}{\partial \alpha_{2}} \mathrm{~F}\left(\alpha_{1}, \alpha_{2}\right)=2 \sum_{\mathrm{i}=1}^{\mathrm{m}}\left(\mathrm{y}_{\mathrm{i}}-\alpha_{1} \mathrm{x}_{\mathrm{i}}-\alpha_{2}\right)=0 .
$$


Da equação (15) resulta

$$
\alpha_{1}=\frac{\mathrm{m} \sum_{\mathrm{k}=1}^{\mathrm{m}} \mathrm{x}_{\mathrm{k}} \mathrm{y}_{\mathrm{k}}-\sum_{\mathrm{k}=1}^{\mathrm{m}} \mathrm{x}_{\mathrm{k}} \sum_{\mathrm{k}=1}^{\mathrm{m}} \mathrm{y}_{\mathrm{k}}}{\mathrm{m} \sum_{\mathrm{k}=1}^{\mathrm{m}} \mathrm{x}_{\mathrm{k}}^{2}-\left(\sum_{\mathrm{k}=1}^{\mathrm{m}} \mathrm{x}_{\mathrm{k}}\right)^{2}} \quad \mathrm{e} \quad \alpha_{2}=\frac{\sum_{\mathrm{k}=1}^{\mathrm{m}} \mathrm{x}_{\mathrm{k}} \mathrm{y}_{\mathrm{k}} \sum_{\mathrm{k}=1}^{\mathrm{m}} \mathrm{x}_{\mathrm{k}}-\sum_{\mathrm{k}=1}^{\mathrm{m}} \mathrm{y}_{\mathrm{k}} \sum_{\mathrm{k}=1}^{\mathrm{m}} \mathrm{x}_{\mathrm{k}}^{2}}{\left(\sum_{\mathrm{k}=1}^{\mathrm{m}} \mathrm{x}_{\mathrm{k}}\right)^{2}-\mathrm{m} \sum_{\mathrm{k}=1}^{\mathrm{m}} \mathrm{x}_{\mathrm{k}}^{2}} .
$$

Assim, obtemos as fórmulas para a regressão linear com essa metodologia [13].

\section{$2^{\text {a }}$ Metodologia}

Usando a notação para o produto interno canônico $\langle\mathrm{x}, \mathrm{y}\rangle$ no $\mathbb{R}^{\mathrm{m}}$, tem-se

$$
\langle\mathrm{x}, \mathrm{y}\rangle=\sum_{\mathrm{k}=1}^{\mathrm{m}} \mathrm{x}_{\mathrm{k}} \cdot \mathrm{y}_{\mathrm{k}}=\mathrm{x}^{\mathrm{T}} \cdot \mathrm{y}
$$

onde $\mathrm{x}=\left(\mathrm{x}_{1}, \ldots, \mathrm{x}_{\mathrm{m}}\right)^{\mathrm{T}}$ e $\mathrm{y}=\left(\mathrm{y}_{1}, \ldots, \mathrm{y}_{\mathrm{m}}\right)^{\mathrm{T}}$. Com o produto matricial dado pelo último termo do lado direito da equação (17), o sistema normal $\mathrm{A} \alpha=\mathbf{b}$ para a regressão linear pode ser escrito na forma

$$
\left(\begin{array}{ll}
\overline{\mathrm{g}_{1}} \mathrm{~T} \cdot \overline{\mathrm{g}_{1}} & \overline{\mathrm{g}_{1}} \mathrm{~T} \cdot \overline{\mathrm{g}_{2}} \\
\overline{\mathrm{g}_{1}} \mathrm{~T} \cdot \overline{\mathrm{g}_{2}} & \overline{\mathrm{g}_{2}} \mathrm{~T} \cdot \overline{\mathrm{g}_{2}}
\end{array}\right)\left(\begin{array}{l}
\alpha_{1} \\
\alpha_{2}
\end{array}\right)=\left(\begin{array}{l}
\overline{\mathrm{f}}^{\mathrm{T}} \cdot \overline{\mathrm{g}_{1}} \\
\overline{\mathrm{f}} \cdot \overline{\mathrm{g}_{2}}
\end{array}\right),
$$

ou ainda na forma

$$
\left(\begin{array}{ll}
\mathrm{a}_{11} & \mathrm{a}_{12} \\
\mathrm{a}_{21} & \mathrm{a}_{22}
\end{array}\right)\left(\begin{array}{l}
\alpha_{1} \\
\alpha_{2}
\end{array}\right)=\left(\begin{array}{l}
\mathrm{b}_{1} \\
\mathrm{~b}_{2}
\end{array}\right)
$$

onde cada elemento $a_{i j}$ e $b_{i}$ dado por

$$
a_{i j}=\left\langle\overline{g_{i}} \overline{g_{j}}\right\rangle=\bar{g}_{i}^{T} \cdot \overline{g_{j}}=\sum_{k=1}^{m} g_{i}\left(x_{k}\right) g_{j}\left(x_{k}\right), \quad \text { e } b_{i}=\left\langle\overline{g_{i}}, \bar{f}\right\rangle=\sum_{k=1}^{m} g_{i}\left(x_{k}\right) f\left(x_{k}\right) .
$$

Usando a regra de Cramer [7] para resolver o sistema (18), tem-se que

$$
\alpha_{1}=\frac{\operatorname{det}\left(\begin{array}{ll}
\overline{\mathrm{f}}^{\mathrm{T}} \cdot \overline{\mathrm{g}_{1}} & \overline{\mathrm{g}}_{1}^{\mathrm{T}} \cdot \overline{\mathrm{g}_{2}} \\
\overline{\mathrm{f}}^{\mathrm{T}} \cdot \overline{\mathrm{g}_{2}} & \overline{\mathrm{g}_{2}} \mathrm{~T} \cdot \overline{\mathrm{g}_{2}}
\end{array}\right)}{\operatorname{det}(\mathrm{A})} \quad \text { e } \quad \alpha_{2}=\frac{\operatorname{det}\left(\begin{array}{ll}
\overline{\mathrm{g}}_{1}^{\mathrm{T}} \cdot \overline{\mathrm{g}_{1}} & \overline{\mathrm{f}}^{\mathrm{T}} \cdot \overline{\mathrm{g}_{1}} \\
\overline{\mathrm{g}}_{1}^{\mathrm{T}} \cdot \overline{\mathrm{g}_{2}} & \overline{\mathrm{f}}^{\mathrm{T}} \cdot \overline{\mathrm{g}_{2}}
\end{array}\right)}{\operatorname{det}(\mathrm{A})}
$$

onde $\operatorname{det}(\mathrm{A})=\left(\overline{\mathrm{g}}_{1}^{\mathrm{T}} \cdot \overline{\mathrm{g}}_{1}\right) \cdot\left(\overline{\mathrm{g}}^{\mathrm{T}} \cdot \overline{\mathrm{g}_{2}}\right)-\left({\overline{\mathrm{g}_{1}}}^{\mathrm{T}} \cdot \overline{\mathrm{g}}_{2}\right)^{2}$ deve ser diferente de zero.

\section{$3^{\text {a }}$ Metodologia}

Esse procedimento é baseado em funções $\mathrm{g}_{1}(\mathrm{x})$ e $\mathrm{g}_{2}(\mathrm{x})$ de modo que os vetores $\overline{\mathrm{g}_{1}}$ e $\overline{\mathrm{g}_{2}}$ sejam ortogonais em $\mathbb{R}^{\mathrm{m}}$. Em tal caso, na equação (10) devemos considerar $\mathrm{g}_{1}(\mathrm{x})=\mathrm{x}-\mathrm{c}$ e $\mathrm{g}_{2}(\mathrm{x})=1$, onde c é uma constante a determinar, mediante a condição de ortogonalidade dada por

$$
\left\langle\overline{\mathrm{g}_{1}}, \overline{\mathrm{g}_{2}}\right\rangle=\overline{\mathrm{g}}_{1}^{\mathrm{T}} \cdot \overline{\mathrm{g}_{2}}=0,
$$


sendo $\overline{g_{1}}=\left(x_{1}-c, x_{2}-c, \ldots, x_{m}-c\right)^{\mathrm{T}}$ e $\overline{g_{2}}=(1,1, \ldots, 1)^{\mathrm{T}}$.

Da equação (22) e dos vetores $\overline{g_{1}}$ e $\overline{g_{2}}$ obtemos o valor da constante c dada por

$$
c=\frac{1}{m} \sum_{i=1}^{m} x_{i}
$$

O sistema matricial $\mathrm{A} \alpha=\mathbf{b}$ dado pela equação (18) reduz-se a

$$
\left(\begin{array}{cc}
\overline{\mathrm{g}}_{1}^{\mathrm{T}} \cdot \overline{\mathrm{g}_{1}} & 0 \\
0 & {\overline{\mathrm{g}_{2}}}^{\mathrm{T}} \cdot \overline{\mathrm{g}_{2}}
\end{array}\right)\left(\begin{array}{l}
\alpha_{1} \\
\alpha_{2}
\end{array}\right)=\left(\begin{array}{l}
\overline{\mathrm{f}}^{\mathrm{T}} \cdot \overline{\mathrm{g}_{1}} \\
\overline{\mathrm{f}}^{\mathrm{T}} \cdot \overline{\mathrm{g}_{2}}
\end{array}\right) .
$$

Resolvendo o sistema diagonal dado na equação (24) obtém-se

$$
\alpha_{1}=\frac{\overline{\mathrm{g}}^{\mathrm{T}} \cdot \overline{\mathrm{f}}}{\overline{\mathrm{g}}^{\mathrm{T}} \cdot \overline{\mathrm{g}_{1}}}=\frac{\sum_{\mathrm{k}=1}^{\mathrm{m}} \mathrm{f}\left(\mathrm{x}_{\mathrm{k}}\right)\left(\mathrm{x}_{\mathrm{k}}-\mathrm{c}\right)}{\sum_{\mathrm{k}=1}^{\mathrm{m}}\left(\mathrm{x}_{\mathrm{k}}-\mathrm{c}\right)^{2}} \text { e } \alpha_{2}=\frac{\overline{\mathrm{g}}^{\mathrm{T}} \cdot \overline{\mathrm{f}}}{\overline{\mathrm{g}}_{2}^{\mathrm{T}} \cdot \overline{\mathrm{g}_{2}}}=\frac{1}{\mathrm{~m}} \sum_{\mathrm{k}=1}^{\mathrm{m}} \mathrm{f}\left(\mathrm{x}_{\mathrm{k}}\right)
$$

Das equações (22), (23) e (24) tem-se a reta da melhor aproximação para f( $\left.\mathrm{x}_{\mathrm{i}}\right)$ pelos mínimos quadrados, dado por

$$
\varphi(\mathrm{x})=\alpha_{1}(\mathrm{x}-\mathrm{c})+\alpha_{2}
$$

Observação: Os parâmetros $\alpha_{1}$ e $\alpha_{2}$ também podem ser obtidos da seguinte forma:

Considere $\bar{\varphi}$ o vetor de $\mathbb{R}^{\mathrm{m}}$ dado por $\bar{\varphi}=\left(\varphi\left(\mathrm{x}_{1}\right), \varphi\left(\mathrm{x}_{2}\right), \ldots \varphi\left(\mathrm{x}_{\mathrm{m}}\right)\right)^{\mathrm{T}}$. Esse vetor pode ser escrito como combinação linear dos vetores $\overline{\mathrm{g}_{1}}$ e $\overline{\mathrm{g}_{2}}$ que geram um subespaço vetorial $\mathrm{S}$ de $\mathbb{R}^{\mathrm{m}}$ e, portanto, $\bar{\varphi}$ pode ser escrito de modo único como uma combinação linear desses vetores do tipo

$$
\bar{\varphi}=\alpha_{1} \overline{\mathrm{g}_{1}}+\alpha_{2} \overline{\mathrm{g}_{2}} \text {. }
$$

Como $\bar{\varphi} \cong \overline{\mathrm{f}}$ pelos mínimos quadrados, pode-se realizar o produto interno [5] na equação (27) pelo vetor $\overline{\mathrm{g}}_{1}^{\mathrm{T}}$ para obter $\alpha_{1}=\frac{\overline{\mathrm{f}} \cdot \overline{\mathrm{g}}^{\mathrm{T}}}{{\overline{\mathrm{g}_{1}}}^{\mathrm{T}} \cdot \overline{\mathrm{g}}_{1}^{\mathrm{T}}}$. De forma análoga obtém-se $\alpha_{2}=\frac{{\overline{g_{2}}}^{\mathrm{T}} \cdot \overline{\mathrm{f}}}{{\overline{\mathrm{g}_{2}}}^{\mathrm{T}} \cdot \overline{\mathrm{g}}_{2}}$. A Figura 2 ilustra a decomposição ortogonal de $\overline{\mathrm{f}}$ sobre o subespaço $\mathrm{S}$ contido no $\mathbb{R}^{\mathrm{m}}$. 


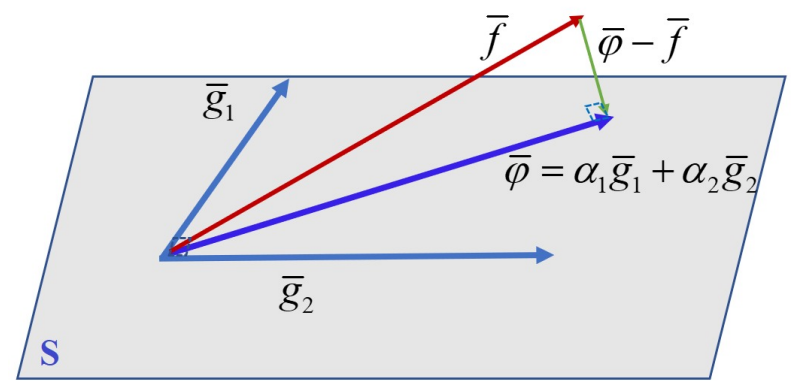

Figura 2: Projeção ortogonal de $\bar{\varphi}$ sobre $\overline{\mathrm{f}}$ no subespaço gerado pelos vetores $\overline{\mathrm{g}_{1}}$ e $\overline{\mathrm{g}_{2}}$

\section{Coeficiente de Determinação}

Uma forma de avaliar a qualidade do ajuste dos pontos experimentais com o método da regressão linear [11] é por meio do coeficiente de determinação $\mathrm{R}^{2}$ dado por [1]

$$
\mathrm{R}^{2}=1-\frac{\sum_{\mathrm{i}=1}^{\mathrm{m}}\left(\mathrm{f}\left(\mathrm{x}_{\mathrm{i}}\right)-\varphi\left(\mathrm{x}_{\mathrm{i}}\right)\right)^{2}}{\sum_{\mathrm{i}=1}^{\mathrm{m}}\left(\mathrm{f}\left(\mathrm{x}_{\mathrm{i}}\right)-\frac{1}{\mathrm{~m}} \sum_{\mathrm{i}=1}^{\mathrm{m}} \mathrm{f}\left(\mathrm{x}_{\mathrm{i}}\right)\right)^{2}} .
$$

A equação (28) mostra que quanto mais próximos estiverem os dados experimentais da reta de ajuste, mais o coeficiente $\mathrm{R}^{2}$ estará próximo da unidade. O ajuste é considerado aceitável quando $0.75<\mathrm{R}^{2} \leq 1$.

\section{Coleta e análise dos exemplares de peixes}

A área litorânea no sul do estado do Rio de Janeiro é caracterizada por apresentar trechos relativamente estreitos de terrenos planos localizados na base da Serra do Mar, muito próxima do oceano [6]. Assim, a encosta leste dessas montanhas, que forma a borda leste do Planalto Brasileiro, é cheia de rios relativamente curtos que geralmente correm no máximo por algumas dezenas de quilômetros até o Oceano Atlântico [3,6]. Sendo esses rios, em geral, pequenos e rasos, os peixes e outros animais aquáticos que neles habitam também são em geral de pequeno porte. Desse modo, poucas são as espécies de peixes que podem ser usadas como alimento pela população ribeirinha que habita essas regiões, e que, por esse motivo, veem tais rios como de pouca importância. Os espécimes utilizados nesse projeto foram coletadas pela equipe do Laboratório de Estudos de Peixes da FFP, de 2007 a 2012 em cinco diferentes pontos da bacia do rio Aldeia (Licença ICMBio 15624-3). O objetivo dessas coletas foi verificar a ocorrência de ictiofauna nos riachos dessa região, fortemente impactada pelas demandas produzidas pelo crescimento urbano desorganizado, fazendo com que a maioria desses corpos d'água se tornassem esgotos a céu aberto. Mesmo assim, ainda é possível observar vida aquática em alguns desses rios [12].

Nas capturas desses peixes foram utilizadas peneiras de malha fina e rede de arrasto de $1,5 \mathrm{~m}$ de comprimento por 1,2 $\mathrm{m}$ de altura com malha de $1 \mathrm{~cm}$ entre-nós. Após anestesia alguns exemplares foram fixados em formalina a 10\%, garantindo o bom estado de conservação de suas vísceras e 
tecidos musculares. São mantidos em álcool $70^{\circ} \mathrm{GL}$, rotulados com dados das coletas e incorporados à Coleção Científica da Uerj - FFP, onde são usados em diversos projetos de pesquisa.

Deuterodon hastatus (Myers, 1928) foi a espécie predominante nos ambientes coletados ao longo dos 5 anos de amostragem. Essa espécie é de pequeno porte, atingindo cerca de 5 a $6 \mathrm{~cm}$ quando adultos. Sua alimentação básica consiste de matéria vegetal e de pequenos artrópodos. Suas populações estão expostas a intensos problemas sazonais relacionados à diminuição da água disponível devido à captação de água para consumo e a intensa poluição na maior parte do curso, o desmatamento das margens dos riachos e assoreamento e aterramento causados pelos projetos de habitações. O isolamento em pequenas ilhas de salubridade certamente levará a fauna aquática dessas áreas à extinção local.

Foram coletados 223 exemplares, e todos eles foram dissecados. Como nessa espécie não há dimorfismo sexual, apenas a observação direta das gônadas permite identificar o sexo dos indivíduos, e foram identificados 130 machos e 93 fêmeas. Para esse trabalho foram avaliadas sete medidas corporais relacionadas ao modo de locomoção e à captura de alimento, duas atividades de grande importância biológica. As medidas são exibidas na Figura 3 e são abreviadas como segue

CP: Comprimento padrão, isto é, o comprimento do exemplar sem a nadadeira caudal;

CT: Comprimento total, incluindo a nadadeira caudal;

CC: Comprimento da cabeça, medido da ponta do focinho até o final do opérculo;

ACb: Altura da cabeça, medida na região do opérculo;

ACo: Altura do corpo medida à frente da dorsal, na região de maior altura corporal;

DO: Diâmetro do olho;

LB: Largura da boca, medida com a boca fechada;

CF: Comprimento do focinho com a boca fechada.

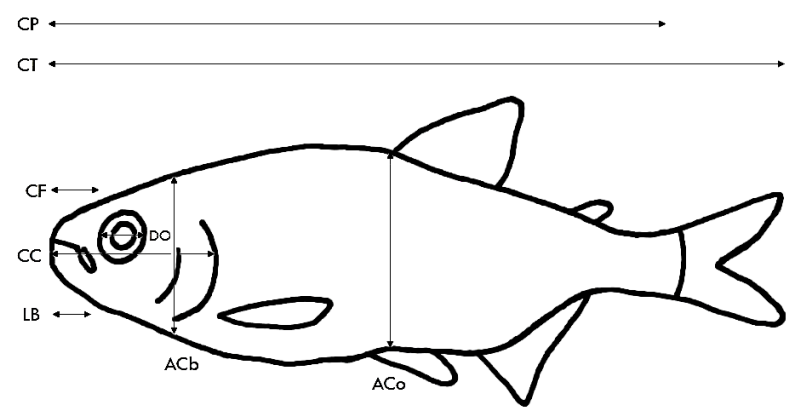

Figura 3: Características merísticas avaliadas em Deuterodon hastatus.

\section{Resultados}

Esta seção traz aplicações de regressão linear para identificar possíveis indicativos de alometrias entre os peixes da espécie Deuterodon hastatus descritas nas seção 5. Na primeira aplicação consideramos apenas seis (6) exemplares desses peixes, onde são estabelecidas certas proporcionalidades entre determinados órgãos dessa espécie animal por meio de três metodologias para a regressão linear. A segunda aplicação envolveu a observação de relação de alometria entre machos e fêmeas 
dessa espécie em relação ao comprimento padrão e o peso do exemplar. Na terceira aplicação são avaliados, sob o ponto de vista da alometria, o comprimento padrão e a altura do corpo medida à frente da nadadeira dorsal. As regressões lineares estabelecidas na segunda e terceira aplicações foram obtidas através da terceira metodologia pela maior facilidade de cálculos.

\subsection{Aplicação 1}

Esse experimento refere-se aos dados do Quadro 1 do lote RSL 2007051502 onde constam somente exemplares fêmeas coletadas em 15/05/2007, conforme metodologia de captura já descrita anteriormente. O objetivo é obter um indicativo de alometria envolvendo o comprimento padrão e o peso do animal.

Consideremos os pontos $\left(\mathrm{x}_{\mathrm{i}}, \mathrm{y}_{\mathrm{i}}\right)$ onde $\mathrm{x}_{\mathrm{i}}$ representa o comprimento padrão e $\mathrm{y}_{\mathrm{i}}$ representa o peso do i-ésimo indivíduo coletado, $\mathrm{i}=1, \ldots, 6$.

Quadro 1: Dados sobre o peso e o comprimento padrão das espécies fêmeas coletadas

\begin{tabular}{|c|c|c|c|c|c|c|}
\hline Número do Exemplar & 1 & 2 & 3 & 4 & 5 & 6 \\
\hline \hline $\mathrm{x}:$ Comprimento Padrão $(\mathrm{mm})$ & 51.96 & 50.05 & 40.35 & 37.59 & 39.27 & 33.99 \\
\hline y : Peso do exemplar $(\mathrm{g})$ & 3.30 & 3.90 & 2.90 & 2.70 & 2.60 & 2.40 \\
\hline
\end{tabular}

Usando os pares (x,y) obtidos do Quadro 1 e pela equação (5), podemos formar novos pares $(\mathrm{X}, \mathrm{Y})$ para formar a Tabela 6.1 dada abaixo por

\begin{tabular}{|c|c|c|c|c|c|c|}
\hline Número do Exemplar & 1 & 2 & 3 & 4 & 5 & 6 \\
\hline $\mathrm{X}=\ln \mathrm{x}$ & 3.9504 & 3.9130 & 3.6976 & 3.6267 & 3.6705 & 3.5261 \\
\hline $\mathrm{Y}=\ln \mathrm{y}$ & 1.1939 & 1.3610 & 1.0647 & 0.9933 & 0.9555 & 0.8755 \\
\hline
\end{tabular}

Tabela 1: Construção dos pares (X, Y).

Com base nos dados apresentados na Tabela 1, é possível estabelecer uma correlação linear entre os pares $(\mathrm{X}, \mathrm{Y})$. Para isso, define-se a função linear $\varphi(\mathrm{X})=\alpha_{1} \mathrm{~g}_{1}(\mathrm{X})+\alpha_{2} \mathrm{~g}_{2}(\mathrm{X})$ por meio das metodologias descritas na $3^{\text {a }}$ seção.

1ª Metodologia: Substituindo os valores da Tabela 6.1 na equação (16) obtemos

$$
\alpha_{1}=\frac{6 \sum_{\mathrm{k}=1}^{6} \mathrm{X}_{\mathrm{k}} \mathrm{Y}_{\mathrm{k}}-\sum_{\mathrm{k}=1}^{6} \mathrm{X}_{\mathrm{k}} \sum_{\mathrm{k}=1}^{6} \mathrm{Y}_{\mathrm{k}}}{6 \sum_{\mathrm{k}=1}^{6} \mathrm{X}_{\mathrm{k}}^{2}-\left(\sum_{\mathrm{k}=1}^{6} \mathrm{X}_{\mathrm{k}}\right)^{2}}=0.9726 \text { e } \alpha_{2}=\frac{\sum_{\mathrm{k}=1}^{6} \mathrm{X}_{\mathrm{k}} \mathrm{Y}_{\mathrm{k}} \sum_{\mathrm{k}=1}^{6} \mathrm{X}_{\mathrm{k}}-\sum_{\mathrm{k}=1}^{6} \mathrm{Y}_{\mathrm{k}} \sum_{\mathrm{k}=1}^{6} \mathrm{X}_{\mathrm{k}}^{2}}{\left(\sum_{\mathrm{k}=1}^{6} \mathrm{X}_{\mathrm{k}}\right)^{2}-6 \sum_{\mathrm{k}=1}^{6} \mathrm{X}_{\mathrm{k}}^{2}}=-2.5546 .
$$

Da equação (29) e da equação (10) tem-se

$$
\varphi(\mathrm{X})=\mathrm{Y}=0.9726 \mathrm{X}-2.5546,
$$

aplicando a função exponencial em ambos os lados da equação (30) e pelas propriedades da função logarítmica, obtém-se a função de alometria dada por

$$
\mathrm{y}=0.077 \mathrm{x}^{0.9726}
$$


isto é, a equação (31) representa a relação entre os crescimentos específicos envolvendo o comprimento padrão e o peso do exemplar.

$\mathbf{2}^{\mathbf{a}}$ Metodologia: A equação (10) pode ser escrita da forma

$$
\mathrm{Y}=\alpha_{1} \mathrm{~g}_{1}(\mathrm{X})+\alpha_{2} \mathrm{~g}_{2}(\mathrm{X})=\alpha_{1} \mathrm{X}+\alpha_{2}
$$

onde $\mathrm{g}_{1}(\mathrm{X})=\mathrm{X}$ e $\mathrm{g}_{2}(\mathrm{X})=1$. Das equações $(11)$ e $(12)$ temos os seguintes vetores

$$
\overline{\mathrm{g}_{1}}=\left(\mathrm{g}_{1}\left(\mathrm{X}_{1}\right), \mathrm{g}_{1}\left(\mathrm{X}_{2}\right), \ldots, \mathrm{g}_{1}\left(\mathrm{X}_{6}\right)\right)^{\mathrm{T}}=\left(\mathrm{X}_{1}, \mathrm{X}_{2}, \ldots, \mathrm{X}_{6}\right)^{\mathrm{T}}=\left(\ln \mathrm{x}_{1}, \ln \mathrm{x}_{2}, \ldots, \ln \mathrm{x}_{6}\right)^{\mathrm{T}}
$$

isto é,

$$
\begin{gathered}
\overline{\mathrm{g}_{1}}=(3.9504,3.9130,3.6976,3.6267,3.6705,3.5261)^{\mathrm{T}} \\
\overline{\mathrm{g}_{2}}=\left(\mathrm{g}_{2}\left(\mathrm{X}_{1}\right), \mathrm{g}_{2}\left(\mathrm{X}_{2}\right), \ldots, \mathrm{g}_{2}\left(\mathrm{X}_{6}\right)\right)^{\mathrm{T}}=(1,1,1,1,1,1)^{\mathrm{T}}
\end{gathered}
$$

$$
\begin{gathered}
\overline{\mathrm{f}}=\left(\mathrm{Y}_{1}, \mathrm{Y}_{2}, \ldots, \mathrm{Y}_{6}\right)^{\mathrm{T}}=\left(\ln \mathrm{y}_{1}, \ln \mathrm{y}_{2}, \ldots, \ln \mathrm{y}_{6}\right)^{\mathrm{T}}= \\
=(1.1939,1.3610,1.0647,0.9933,0.9555,0.8755)^{\mathrm{T}} .
\end{gathered}
$$

Da equação (25) e dos vetores $\overline{g_{1}}, \overline{g_{2}}$ e $\bar{f} \in \mathbb{R}^{6}$ e obtidos das equações (11) e (12) obtêm-se os coeficientes da matriz normal do sistema

$$
\begin{gathered}
\mathrm{a}_{11}=\left\langle\overline{\mathrm{g}_{1}}, \overline{\mathrm{g}_{1}}\right\rangle={\overline{\mathrm{g}_{1}}}^{\mathrm{T}} \cdot \overline{\mathrm{g}_{1}}=\sum_{\mathrm{k}=1}^{6} \mathrm{~g}_{1}\left(\mathrm{X}_{\mathrm{k}}\right) \mathrm{g}_{1}\left(\mathrm{X}_{\mathrm{k}}\right)=83.6488 \\
\mathrm{a}_{12}=\left\langle\overline{\mathrm{g}_{1}}, \overline{\mathrm{g}_{2}}\right\rangle={\overline{\mathrm{g}_{1}}}^{\mathrm{T}} \cdot \overline{\mathrm{g}_{2}}=\sum_{\mathrm{k}=1}^{6} \mathrm{~g}_{1}\left(\mathrm{X}_{\mathrm{k}}\right) \mathrm{g}_{2}\left(\mathrm{X}_{\mathrm{k}}\right)=22.3843 \\
\mathrm{a}_{22}=\left\langle\overline{\mathrm{g}_{2}}, \overline{\mathrm{g}_{2}}\right\rangle={\overline{\mathrm{g}_{2}}}^{\mathrm{T}} \cdot \overline{\mathrm{g}_{2}}=\sum_{\mathrm{k}=1}^{6} \mathrm{~g}_{2}\left(\mathrm{X}_{\mathrm{k}}\right) \mathrm{g}_{2}\left(\mathrm{X}_{\mathrm{k}}\right)=6 \\
\mathrm{~b}_{1}=\left\langle\overline{\mathrm{g}_{1}}, \overline{\mathrm{f}}\right\rangle=\sum_{\mathrm{k}=1}^{6} \mathrm{~g}_{1}\left(\mathrm{X}_{\mathrm{k}}\right) \mathrm{f}\left(\mathrm{X}_{\mathrm{k}}\right)=24.1753 \\
\mathrm{~b}_{2}=\left\langle\overline{\mathrm{g}_{2}}, \overline{\mathrm{f}}\right\rangle=\sum_{\mathrm{k}=1}^{6} \mathrm{~g}_{2}\left(\mathrm{X}_{\mathrm{k}}\right) \mathrm{f}\left(\mathrm{X}_{\mathrm{k}}\right)=6.4438
\end{gathered}
$$

Substituindo esses coeficientes no sistema (18) resulta a equação matricial dada por

$$
\left(\begin{array}{cc}
83.6488 & 22.3843 \\
22.3843 & 6
\end{array}\right)\left(\begin{array}{l}
\alpha_{1} \\
\alpha_{2}
\end{array}\right)=\left(\begin{array}{c}
24.1753 \\
6.4438
\end{array}\right)
$$

A equação (33) pode ser resolvida, por exemplo, pelo método de Cramer dado pela equação (21) obtemos os valores de $\alpha_{1}$ e $\alpha_{2}$, 


$$
\alpha_{1}=\frac{\left|\begin{array}{cc}
24.1753 & 22.3843 \\
6.4438 & 6
\end{array}\right|}{\left|\begin{array}{cc}
83.6488 & 22.3843 \\
22.3843 & 6
\end{array}\right|}=0.9726 \text { e } \alpha_{2}=\frac{\left|\begin{array}{cc}
83.6488 & 24.1753 \\
22.3843 & 6.4438
\end{array}\right|}{\left|\begin{array}{cc}
83.6488 & 22.3843 \\
22.3843 & 6
\end{array}\right|}=-2.5546 .
$$

Das equações (10) e (34) tem-se

$$
\mathrm{Y}=\alpha_{1} \mathrm{X}+\alpha_{2}=0.9726 \mathrm{X}-2.5546 .
$$

Da equação (35) pode ser obtida, usando propriedades básicas do cálculo de logaritmos, a relação alométrica dada por

$$
\mathrm{y}=\mathrm{e}^{\alpha_{2}} \mathrm{x}^{\alpha_{1}}=0.0777 \mathrm{x}^{0.9726} .
$$

$3^{\mathbf{a}}$ Metodologia: Considere as funções $\mathrm{g}_{1}(\mathrm{X})=\mathrm{X}-\mathrm{C}$ e $\mathrm{g}_{2}(\mathrm{X})=1$, onde $\mathrm{C}$ é uma constante a ser determinada pela relação de ortogonalidade entre vetores $\overline{g_{1}}$ e $\overline{g_{2}}$ do espaço $\mathbb{R}^{6}$. Sendo $\overline{\mathrm{g}_{1}}=\left(\mathrm{X}_{1}-\mathrm{C}, \mathrm{X}_{2}-\mathrm{C}, \ldots, \mathrm{X}_{6}-\mathrm{C}\right)^{\mathrm{T}}$ e $\overline{\mathrm{g}_{2}}=(1,1,1,1,1,1)^{\mathrm{T}}$, através da equação $(23)$, obtemos o valor da constante $\mathrm{C}$ dado por $\mathrm{C}=\frac{1}{6} \sum_{\mathrm{i}=1}^{6} \mathrm{X}_{\mathrm{i}}=3.7307$. Como $\overline{\mathrm{g}_{1}}$ e $\overline{\mathrm{g}_{2}}$ são vetores ortogonais no espaço $\mathbb{R}^{6}$, o sistema matricial $\mathrm{A} \alpha=\mathbf{b}$ dado pela equação (24) reduz-se a

$$
\left(\begin{array}{cc}
0.13895 & 0 \\
0 & 6
\end{array}\right)\left(\begin{array}{l}
\alpha_{1} \\
\alpha_{2}
\end{array}\right)=\left(\begin{array}{c}
0.1351 \\
6.4438
\end{array}\right)
$$

Resolvendo o sistema (37) obtém-se

$$
\alpha_{1}=\frac{0.1351}{0.1389}=0.9726 \text { e } \alpha_{2}=\frac{6.4438}{6}=1.074 .
$$

O ajuste linear é dado pela equação (26) com os valores obtidos de $\alpha_{1}, \alpha_{2}$ e C dados pela equação $(38)$.

$$
\mathrm{Y}=0.9726(\mathrm{X}-3.7307)+1.0740=0.9726 \mathrm{X}-2.5546 \text {. }
$$

Da equação (39), usando propriedades básicas do cálculo de logaritmos pode ser obtida a equação de alometria

$$
\mathrm{y}=\mathrm{e}^{-2.5546} \mathrm{x}^{0.9726}=0.0777 \mathrm{x}^{0.9726} .
$$

É possível observar que a terceira metodologia usando a projeção ortogonal de vetores é a mais prática e de fácil manipulação. Entretanto, não é a mais usual, pois ela envolve conceitos de Álgebra Linear básica, os quais não são usuais em livros didáticos sobre o assunto, como [13] em livro escrito para estudantes de Estatística.

Temos que enfatizar que devido ao pequeno número de exemplares escolhidos para a aplicação do método de regressão linear, a alometria obtida não deve ser considerada como determinante para essa espécie de peixe, mas sim como um forte indício de que deve existir essa correlação entre os crescimentos dos órgãos observados para animais dessa espécie.

Através da equação (28) obtemos o coeficiente de determinação $\mathrm{R}^{2}=0.80$, o qual mostra que o ajuste dos pontos observado na Tabela 6.1 é de boa qualidade, conforme pode ser visto na Figura 4, exceto pelo maior desvio ocorrido no ponto relacionado ao quinto indivíduo. 


\begin{tabular}{|c|c|c|c|c|}
\hline Peixes Coletados 15/05/2007 & $\mathrm{a}$ & $\mathrm{b}$ & $\mathrm{R}^{2}$ & Relação Alométrica \\
\hline \hline Peixes fêmeas $(\mathrm{m}=6)$ & 0.077 & 0.9726 & 0.80 & $\mathrm{y}=0.077 \mathrm{x}^{0.9726}$ \\
\hline
\end{tabular}

Tabela 2: Indicativos de relações alométricas de exemplares fêmeas de Deuterodon hastatus em relação ao comprimento padrão e peso do exemplar.

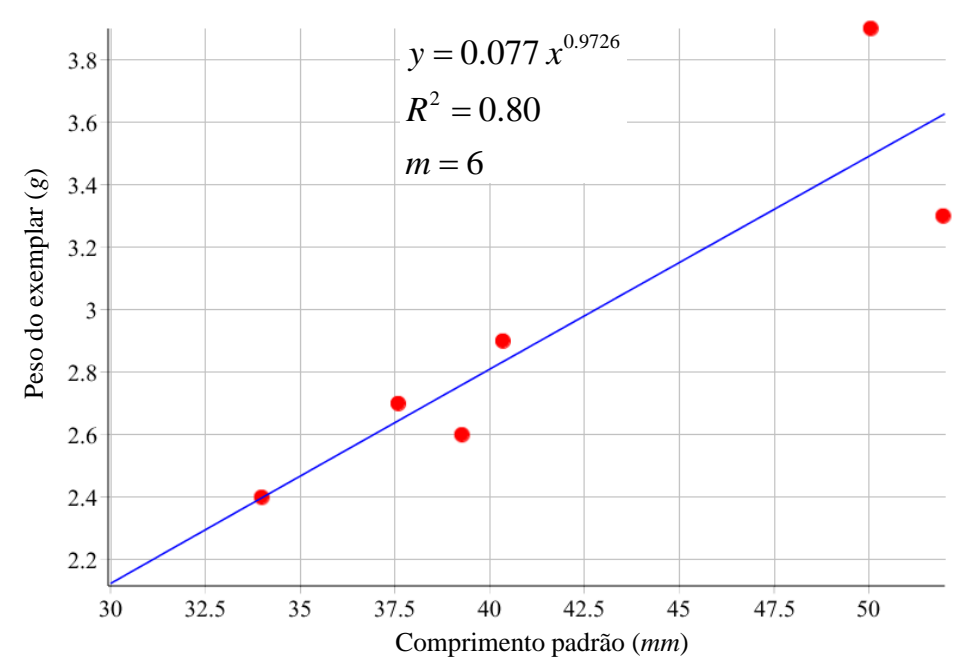

Figura 4: Regressão linear estabelecida entre o peso do exemplar e o comprimento padrão nos exemplares de Deuterodon hastatus.

Nas aplicações que se seguem, os resultados foram determinados através da terceira metodologia por motivos de natureza prática. Os indicativos de alometria são obtidos em relação aos cento e trinta (130) exemplares machos e noventa e três (93) exemplares fêmeas, coletados entre os anos de 2007/2012, diferenciando os exemplares pelo sexo.

Da equação (23) e da equação (25) correspondente à terceira metodologia, tem-se

$$
\mathrm{C}=\frac{1}{\mathrm{~m}} \sum_{\mathrm{i}=1}^{\mathrm{m}} \mathrm{X}_{\mathrm{i}}, \quad \alpha_{1}=\frac{\overline{\mathrm{g}}^{\mathrm{T}} \cdot \overline{\mathrm{f}}}{{\overline{\mathrm{g}_{1}}}^{\mathrm{T}} \cdot \overline{\mathrm{g}_{1}}}=\frac{\sum_{\mathrm{k}=1}^{\mathrm{m}} \mathrm{f}\left(\mathrm{X}_{\mathrm{k}}\right)\left(\mathrm{X}_{\mathrm{k}}-\mathrm{C}\right)}{\sum_{\mathrm{i}=1}^{\mathrm{m}}\left(\mathrm{X}_{\mathrm{k}}-\mathrm{C}\right)^{2}} \text { e } \alpha_{2}=\frac{\overline{\mathrm{g}}^{\mathrm{T}} \cdot \overline{\mathrm{f}}}{{\overline{\mathrm{g}_{2}}}^{\mathrm{T}} \cdot \overline{\mathrm{g}_{2}}}=\frac{1}{\mathrm{~m}} \sum_{\mathrm{k}=1}^{\mathrm{m}} \mathrm{f}\left(\mathrm{X}_{\mathrm{i}}\right) .
$$

onde $\mathrm{m}$ é o número de exemplares de cada sexo. $\mathrm{A}$ variável $\mathrm{X}=\ln (\mathrm{x})$, onde $\mathrm{x}$ representa o comprimento padrão e $\mathrm{Y}=\ln (\mathrm{y})$, como y indicando a altura do corpo medida à frente da dorsal. $\mathrm{Na}$ equação (26) temos a equação linear dada por

$$
\varphi(\mathrm{X})=\alpha_{1}(\mathrm{X}-\mathrm{C})+\alpha_{2}
$$

Das equações (7), (8) e (42) será obtida a relação alométrica.

Devido ao grande número de dados envolvidos nas aplicações 2 e 3, omitiremos nesse desenvolvimento, por razões de práticas, os dados dos vetores $\overline{g_{1}}, \overline{g_{2}}$ e $\bar{f}$, bem como, os valores relativos aos parâmetros envolvidos, objetivo dessa investigação. Os dados experimentais das aplicações 2 e 3 encontram-se no Anexo. 


\subsection{Aplicação 2}

Nesta aplicação obtém-se um indicativo de alometria entre o comprimento padrão e o peso do exemplar, considerando machos e fêmeas.

\section{Analisando os exemplares machos:}

Utilizando a equação (23), temos $\mathrm{C}=3.2104$ enquanto da equação (24) resulta o sistema linear dado

$$
\left(\begin{array}{cc}
7.7442 & 0 \\
0 & 130
\end{array}\right)\left(\begin{array}{l}
\alpha_{1} \\
\alpha_{2}
\end{array}\right)=\left(\begin{array}{c}
20.8704 \\
-97.0620
\end{array}\right) .
$$

Resolvendo o sistema (43) obtêm-se os valores para $\alpha_{1}$ e $\alpha_{2}$ na forma

$$
\alpha_{1}=2.6950 \quad \text { e } \quad \alpha_{2}=-0.7466 .
$$

Da equação (42) obtêm-se

$$
\mathrm{Y}=2.6950(\mathrm{X}-3.2104)-0.7466
$$

ou ainda

$$
\mathrm{Y}=2.6950 \mathrm{X}-9.6764 \text {. }
$$

Da equação (46) obtemos a função de alometria dada por

$$
\mathrm{y}=0.63 \cdot 10^{-4} \mathrm{x}^{2.6950} .
$$

O coeficiente de determinação pode ser obtido por meio da equação (28), isto é,

$$
\mathrm{R}^{2}=0.8482 \text {. }
$$

\section{Analisando os exemplares fêmeas:}

Utilizando a equação (23), temos $C=3.4810$ e da equação (24), tem-se o sistema

$$
\left(\begin{array}{cc}
5.2671 & 0 \\
0 & 93
\end{array}\right)\left(\begin{array}{l}
\alpha_{1} \\
\alpha_{2}
\end{array}\right)=\left(\begin{array}{c}
15.2648 \\
-17.8890
\end{array}\right) .
$$

Resolvendo o sistema (49) obtém-se os valores para $\alpha_{1}$ e $\alpha_{2}$ na forma

$$
\alpha_{1}=2.8981 \quad \text { e } \quad \alpha_{2}=-0.1924 \text {. }
$$

Da equação (42) obtêm-se

$$
\mathrm{Y}=2.8981(\mathrm{X}-3.4810)-0.1924
$$

ou ainda

$$
\mathrm{Y}=2.8981 \mathrm{X}-10.2807
$$

Da equação (52) obtemos a função de alometria dada por

$$
\mathrm{y}=0.34 \cdot 10^{-4} \mathrm{x}^{2.8981} \text {. }
$$


Da equação (28), pode-se obter o coeficiente de determinação dado por

$$
\mathrm{R}^{2}=0.8545
$$

Na Figura 5, encontram-se os gráficos das relações de proporcionalidade envolvendo os exemplares de ambos os sexos.
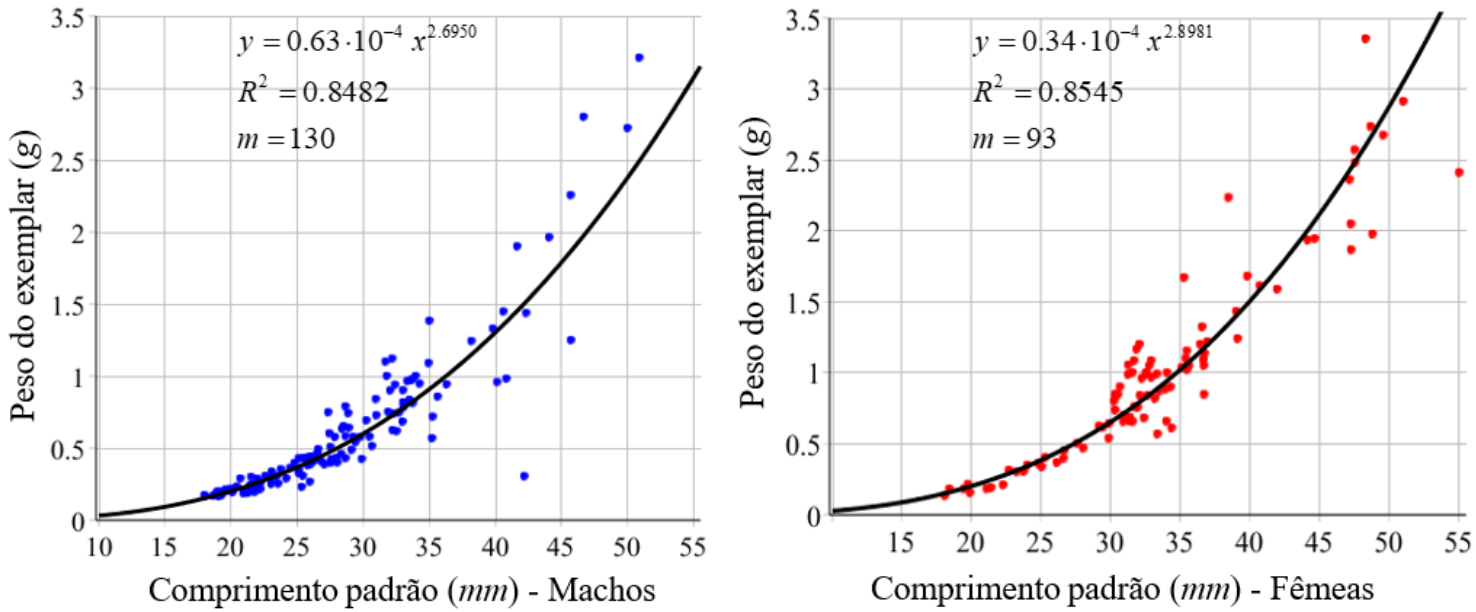

Figura 5: Regressão linear estabelecida entre o comprimento padrão e o peso do exemplar

Na Tabela 6.2 são apresentados os coeficientes da relação alométrica para machos e fêmeas, o coeficiente de determinação e as relações alométricas, respectivamente. Comparando os coeficientes alométricos da Tabela 3, conclui-se que as fêmeas têm massa corporal superior aos machos.

\begin{tabular}{|c|c|c|c|c|}
\hline Peixes Coletados 2007-2012 & $\mathrm{a}$ & $\mathrm{b}$ & $\mathrm{R}^{2}$ & Relação Alométrica \\
\hline \hline Machos $(\mathrm{m}=130)$ & $0.63 \cdot 10^{-4}$ & 2.6950 & 0.8482 & $\mathrm{y}=0.63 \cdot 10^{-4} \mathrm{x}^{2.6950}$ \\
\hline \hline Fêmeas $(\mathrm{m}=93)$ & $0.34 \cdot 10^{-4}$ & 2.8981 & 0.8545 & $\mathrm{y}=0.34 \cdot 10^{-4} \mathrm{x}^{2.8981}$ \\
\hline
\end{tabular}

Tabela 3: Indicativos de relações alométricas de exemplares machos e fêmeas de Deuterodon hastatus em relação ao comprimento padrão e peso do exemplar.

\subsection{Aplicação 3}

O objetivo dessa aplicação é obter um indicativo de alometria entre o comprimento padrão e a altura do corpo medida à frente da dorsal.

\section{Analisando os exemplares machos:}

Considerando os dados do comprimento padrão e da altura do corpo medida à frente da dorsal dos 130 exemplares machos, o sistema $\mathrm{A} \alpha=\mathbf{b}$ dado pela equação (24) reduz-se a

$$
\left(\begin{array}{cc}
7.7442 & 0 \\
0 & 130
\end{array}\right)\left(\begin{array}{l}
\alpha_{1} \\
\alpha_{2}
\end{array}\right)=\left(\begin{array}{c}
9.6389 \\
292.9736
\end{array}\right) .
$$


Da equação (23), obtemos $\mathrm{C}=\frac{1}{130} \sum_{\mathrm{i}=1}^{130} \mathrm{X}_{\mathrm{i}}=3.3125$. Resolvendo o sistema (55) obtêm-se os valores $\alpha_{1}=1.24467$ e $\alpha_{2}=2.253643$; logo, substituindo estes valores na equação (26), tem-se

$$
\mathrm{Y}=1.2447(\mathrm{X}-3.3125)+2.2536
$$

ou ainda

$$
\mathrm{Y}=1.2447 \mathrm{X}-1.86933 .
$$

Da equação (57) pode ser obtida, usando propriedades básicas do cálculo de logaritmos, a relação alométrica dada por

$$
\mathrm{y}=\mathrm{e}^{-1.8693} \mathrm{x}^{1.2447}
$$

ou ainda

$$
\mathrm{y}=0.1542 \mathrm{x}^{1.2447} .
$$

E da equação (28) o coeficiente de determinação $\mathrm{R}^{2}=0.9222$.

\section{Analisando os exemplares fêmeas:}

Considerando os dados do comprimento padrão e da altura do corpo medida à frente da dorsal dos 93 exemplares fêmeas, o sistema matricial A $\alpha=\mathbf{b}$ dado pela equação (24) reduz-se a

$$
\left(\begin{array}{cc}
5.2671 & 0 \\
0 & 93
\end{array}\right)\left(\begin{array}{l}
\alpha_{1} \\
\alpha_{2}
\end{array}\right)=\left(\begin{array}{c}
7.2189 \\
232.3256
\end{array}\right) .
$$

Resolvendo o sistema (60) obtém-se $\alpha_{1}=1.3706$ e $\alpha_{2}=2.4981$; da equação (23) obtém-se $\mathrm{C}=$ $\frac{1}{93} \sum_{i=1}^{93} \mathrm{X}_{\mathrm{i}}=3.3125$

Substituindo os valores de $\alpha_{1}, \alpha_{2}$ e C na equação (26), tem-se

$$
\mathrm{Y}=1.3706(\mathrm{X}-3.481)+2.4981
$$

ou ainda

$$
\mathrm{Y}=1.3706 \mathrm{X}-2.273 \text {. }
$$

Da equação (62) pode ser obtida, usando propriedades básicas do cálculo de logaritmos, a relação alométrica

$$
\mathrm{y}=\mathrm{e}^{-2.273} \mathrm{x}^{1.3706}
$$

ou ainda

$$
\mathrm{y}=0.103 \mathrm{x}^{1.2447} \text {. }
$$

E da equação (28) o coeficiente de determinação $\mathrm{R}^{2}=0.9122$.

Na Figura 6, encontram-se o gráfico da relação de proporcionalidade entre o comprimento padrão e a altura do corpo medida à frente da dorsal dos 93 exemplares fêmeas do peixe Deuterodon hastatus. 

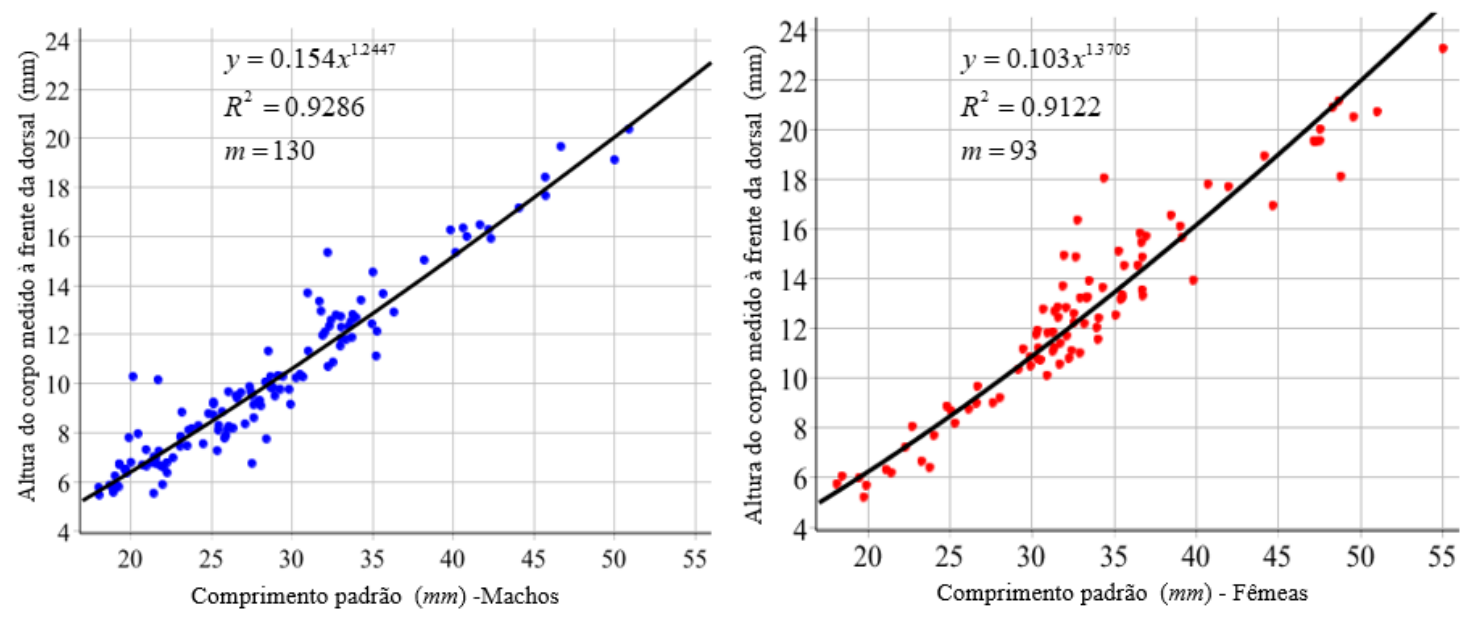

Figura 6: Regressão linear entre o comprimento padrão e a altura do corpo medido à frente da dorsal de exemplares machos e fêmeas Deuterodon hastatus

Na Tabela 6.3 são apresentados os coeficientes da relação alométrica para machos e fêmeas, o coeficiente de determinação e as relações alométricas respectivamente. Comparando os coeficientes alométricos nessa tabela, observa-se que o coeficiente alométrico da fêmea é um pouco maior do que o coeficiente alométrico dos machos, o que leva a concluir que as fêmeas têm altura corporal ligeiramente maior do que a altura corporal dos machos.

\begin{tabular}{|c|c|c|c|c|}
\hline Peixes Coletados 2007-2012 & $\mathrm{a}$ & $\mathrm{b}$ & $\mathrm{R}^{2}$ & Relação Alométrica \\
\hline \hline Machos $(\mathrm{m}=130)$ & 0.1540 & 1.2447 & 0.9222 & $\mathrm{y}=0.1504 \mathrm{x}^{1.2447}$ \\
\hline Fêmeas $(\mathrm{m}=93)$ & 0.1030 & 1.3706 & 0.9122 & $\mathrm{y}=0.1030 \mathrm{x}^{1.3706}$ \\
\hline
\end{tabular}

Tabela 4: Indicativos de relações alométricas de exemplares machos e fêmeas de Deuterodon hastatus em relação ao comprimento padrão e à altura do corpo medida à frente da dorsal.

Avaliações alométricas permitem observar se existe relação de proporcionalidade entre dois diferentes órgãos de um mesmo indivíduo de uma determinada espécie animal. Nesse caso, foi particularizado o indicativo de relação entre as medidas envolvendo o peso e o comprimento padrão. Entretanto, ao analisar outras possíveis indicações de alometria, observou-se que a altura da cabeça cresceu 4 vezes menos que a altura do corpo, e isso leva a pensar que é uma vantagem para o peixe ter a extremidade anterior mais afunilada, assim diminuindo o atrito com a água.

Os exemplares dessa espécie, como da maioria dos peixes, realizam fecundação externa, isto é, os gametas são jogados na água, onde se encontram ao acaso para formar o zigoto. Nos animais que realizam esse tipo de fecundação há uma alta taxa de produção de gametas, pois quanto mais gametas estiverem na água, maior a chance de encontro de ovócitos e espermatozoides. Assim, as fêmeas normalmente são maiores e mais largas devido ao fato de possuírem grandes ovócitos que necessitam de mais espaço para serem armazenados, enquanto os espermatozoides são células de menor porte. A diferença nessas proporções possivelmente confere maior eficácia ao nado dos machos para fuga de predadores, bem como a captura de alimento. 


\subsection{Conclusões}

Neste estudo o método de regressão linear foi utilizado como um indicativo de alometria envolvendo comprimento padrão e peso do exemplar, bem como o comprimento padrão e altura do corpo medida à frente da dorsal na espécie de peixe Deuterodon hastatus. Uma das metodologias aqui utilizadas para a obtenção desses indicativos de alometria foi a projeção ortogonal de vetores. Esse procedimento destaca-se pela facilidade de cálculos para a obtenção dos parâmetros envolvidos no modelo alométrico. Entretanto, essa técnica necessita de aspectos básicos de Álgebra Linear, a qual não é comumente adotada nos cursos de licenciatura de áreas como Estatística, Biologia e Química.

A primeira aplicação envolveu um pequeno lote de exemplares dessa espécie de peixe, o que possibilitou descrever com detalhes todas as etapas com respeito às metodologias aqui utilizadas para a regressão linear, correlacionando os parâmetros de comprimento padrão e o peso do exemplar. Com respeito às demais aplicações, cada regressão linear foi obtida com a terceira metodologia devido a sua maior praticidade de cálculos. Entretanto, devido ao elevado número de exemplares, não foram apresentados explicitamente, os vetores que dão origem a cada sistema linear correspondente e que derivam dos parâmetros de crescimento dos órgãos de interesse, e que por esse motivo, constam os dados tabelados em Anexo.

Em relação a segunda aplicação, um indicativo de alometria foi determinado entre o comprimento padrão e o peso do exemplar. Na terceira aplicação, a relação alométrica foi entre o comprimento padrão e a altura da cabeça medida à frente da dorsal. Esses indicativos de proporcionalidade existentes entre essas partes desses animais mostram que as fêmeas possuem maior massa corporal, enquanto os machos são mais esguios que as fêmeas.

O método dos mínimos quadrados lineares apresentado em sua forma mais elementar pode ser usado pelo professor de matemática e/ou biologia no Ensino Médio, como uma alternativa para o ensino e aprendizagem de problemas interdisciplinares de forma contextualizada. A primeira metodologia requer apenas conhecimentos da equação da reta (função polinomial do primeiro grau) e sistemas lineares com duas variáveis. No presente trabalho, onde se objetivou a obtenção de indicativos de alometria entre alguns orgãos dos peixes da espécie analisada, o conhecimento de funções logarítmicas faz-se necessário. Portanto, esse método pode ser usado por um professor em sala de aula para estabelecer correlações ou não, entre grandezas de interesse prático.

Como continuidade de nossa pesquisa pretendemos verificar a existência de outros indicativos alométricos dessa espécie de peixe, considerando diferentes graus de maturação gonadal, e também verificar possíveis aspectos ontogênicos decorrentes desses estudos.

\section{Anexo}

Dados em relação ao Comprimento padrão ( $\mathrm{mm})$, Peso do exemplar (g) e Comprimento da cabeça medida à frente da dorsal $(\mathrm{mm})$ dos 130 exemplares machos e dos 93 exemplares fêmeas. 
Tabela 5: Comprimento padrão dos exemplares machos

\begin{tabular}{|l|l|l|l|l|l|l|l|l|l|}
\hline 32.21 & 40.62 & 22.24 & 39.83 & 40.15 & 33.52 & 29.43 & 24.82 & 33.03 & 18.69 \\
\hline 34.96 & 44.08 & 23.59 & 42.34 & 40.85 & 33.72 & 32.32 & 25.67 & 33.04 & 19.02 \\
\hline 41.66 & 45.71 & 25.45 & 27.37 & 45.73 & 34.28 & 32.42 & 26.06 & 20.01 & 19.09 \\
\hline 19.05 & 20.14 & 25.98 & 28.67 & 21.42 & 21.74 & 33.06 & 26.35 & 21.43 & 20.97 \\
\hline 19.25 & 20.45 & 27.52 & 28.88 & 22.25 & 27.62 & 50.01 & 26.53 & 21.83 & 21.46 \\
\hline 25.81 & 21.69 & 27.63 & 29.14 & 24.49 & 28.00 & 18.91 & 26.61 & 23.06 & 21.54 \\
\hline 27.09 & 21.97 & 28.35 & 35.22 & 25.36 & 28.07 & 18.92 & 27.45 & 31.70 & 22.03 \\
\hline 31.90 & 23.17 & 28.68 & 35.65 & 25.89 & 32.99 & 19.62 & 27.53 & 31.80 & 22.62 \\
\hline 35.02 & 19.29 & 28.94 & 42.20 & 26.08 & 33.77 & 19.74 & 27.87 & 32.05 & 23.50 \\
\hline 50.90 & 19.29 & 29.82 & 26.84 & 33.35 & 36.32 & 20.72 & 28.68 & 33.98 & 23.79 \\
\hline 21.27 & 19.68 & 30.68 & 30.50 & 28.53 & 24.20 & 21.55 & 29.27 & 46.68 & 25.43 \\
\hline 32.23 & 19.88 & 32.55 & 31.01 & 30.97 & 25.13 & 23.09 & 29.91 & 18.01 & 25.93 \\
\hline 38.20 & 20.95 & 35.27 & 33.65 & 32.73 & 25.93 & 23.13 & 30.25 & 18.04 & 28.41 \\
\hline
\end{tabular}

Tabela 6: Peso dos exemplares machos

\begin{tabular}{|l|l|l|l|l|l|l|l|l|l|}
\hline 1.1205 & 1.4482 & 0.2145 & 1.3294 & 0.9580 & 0.8347 & 0.5407 & 0.3960 & 0.9010 & 0.1700 \\
\hline 1.0900 & 1.9633 & 0.2531 & 1.4376 & 0.9820 & 0.9770 & 0.7388 & 0.4320 & 0.8180 & 0.1987 \\
\hline 1.9005 & 2.2562 & 0.3067 & 0.7480 & 1.2490 & 0.9472 & 0.9388 & 0.3878 & 0.2175 & 0.1659 \\
\hline 1.1808 & 0.1931 & 0.2653 & 0.7890 & 0.2113 & 0.2157 & 0.7825 & 0.4140 & 0.1972 & 0.2048 \\
\hline 0.1744 & 0.2324 & 0.4002 & 0.7420 & 0.2567 & 0.4244 & 2.7216 & 0.4620 & 0.1951 & 0.2408 \\
\hline 0.3806 & 0.2488 & 0.4020 & 0.4870 & 0.3620 & 0.4317 & 0.1740 & 0.4930 & 0.2507 & 0.2992 \\
\hline 0.3862 & 0.2480 & 0.4588 & 0.5680 & 0.2298 & 0.3997 & 0.1888 & 0.6020 & 1.1000 & 0.2865 \\
\hline 0.7515 & 0.2947 & 0.4291 & 0.8570 & 0.4373 & 0.6833 & 0.2130 & 0.5070 & 1.0000 & 0.3063 \\
\hline 1.3837 & 0.1718 & 0.6403 & 0.3040 & 0.4412 & 0.8121 & 0.2060 & 0.5760 & 0.9000 & 0.3048 \\
\hline 3.2104 & 0.1967 & 0.5775 & 0.4079 & 0.9654 & 0.9424 & 0.2880 & 0.5820 & 1.0000 & 0.3522 \\
\hline 0.1888 & 0.2028 & 0.5135 & 0.5788 & 0.6520 & 0.2892 & 0.2340 & 0.5780 & 2.8000 & 0.4298 \\
\hline 0.6245 & 0.2116 & 0.6174 & 0.7270 & 0.8399 & 0.3487 & 0.3360 & 0.4240 & 0.1728 & 0.4287 \\
\hline 1.2430 & 0.1859 & 0.7175 & 0.9720 & 0.7465 & 0.3262 & 0.3020 & 0.6920 & 0.1636 & 0.6341 \\
\hline
\end{tabular}

Tabela 7: Altura do corpo medida à frente da dorsal dos exemplares machos

\begin{tabular}{|c|c|c|c|c|c|c|c|c|c|}
\hline 15.35 & 16.35 & 6.78 & 16.27 & 15.35 & 12.34 & 10.30 & 8.78 & 12.74 & 5.86 \\
\hline 12.44 & 17.17 & 8.11 & 15.92 & 16.00 & 11.89 & 12.35 & 8.86 & 11.84 & 6.24 \\
\hline 16.48 & 18.42 & 8.30 & 9.88 & 17.67 & 13.41 & 12.59 & 9.67 & 6.79 & 5.90 \\
\hline 5.79 & 10.29 & 8.12 & 10.29 & 5.53 & 7.25 & 12.30 & 8.19 & 7.02 & 6.64 \\
\hline 5.80 & 7.96 & 6.75 & 9.80 & 6.37 & 9.14 & 19.93 & 9.51 & 6.66 & 6.86 \\
\hline 7.78 & 10.16 & 8.61 & 10.33 & 7.55 & 9.32 & 5.57 & 9.40 & 7.45 & 6.75 \\
\hline 8.36 & 5.89 & 10.07 & 11.93 & 7.27 & 9.10 & 5.66 & 9.67 & 13.35 & 6.59 \\
\hline 11.97 & 8.84 & 9.84 & 13.67 & 7.88 & 11.54 & 6.50 & 9.51 & 12.96 & 6.98 \\
\hline 14.55 & 6.73 & 9.50 & 16.29 & 8.26 & 12.82 & 6.36 & 9.23 & 12.11 & 7.74 \\
\hline 20.38 & 6.70 & 9.77 & 9.64 & 11.80 & 12.92 & 6.69 & 9.97 & 12.69 & 8.17 \\
\hline 6.80 & 6.52 & 10.28 & 10.38 & 11.33 & 8.29 & 6.97 & 9.77 & 19.67 & 8.10 \\
\hline 10.70 & 7.80 & 10.88 & 11.33 & 13.70 & 9.17 & 7.85 & 9.16 & 5.77 & 8.73 \\
\hline 15.04 & 7.31 & 12.14 & 12.53 & 12.80 & 9.25 & 7.77 & 10.24 & 5.46 & 7.75 \\
\hline
\end{tabular}


Tabela 8: Comprimento padrão dos exemplares fêmeas

\begin{tabular}{|c|c|c|c|c|c|c|c|c|c|}
\hline 39.02 & 36.7 & 49.58 & 30.32 & 32.11 & 24.02 & 23.76 & 36.57 & 18.42 & 34.05 \\
\hline 48.8 & 31.87 & 44.67 & 30.52 & 36.71 & 24.8 & 30.91 & 26.67 & 29.16 & 34.3 \\
\hline 31.59 & 35.27 & 35.61 & 31.26 & 29.45 & 25.3 & 31.94 & 30.32 & 29.9 & 35.39 \\
\hline 18.1 & 38.46 & 36.66 & 31.27 & 39.12 & 27.61 & 32.66 & 31.34 & 35.09 & \\
\hline 19.47 & 48.31 & 36.94 & 31.36 & 47.27 & 30.25 & 32.76 & 31.71 & 48.67 & \\
\hline 26.14 & 29.86 & 39.82 & 31.68 & 55.03 & 30.37 & 32.93 & 33.18 & 25.05 & \\
\hline 26.61 & 44.15 & 40.71 & 32.54 & 19.9 & 30.94 & 33.47 & 32.4 & 30.67 & \\
\hline 28.03 & 47.16 & 41.96 & 32.91 & 21.1 & 19.75 & 35.47 & 33.33 & 31.56 & \\
\hline 32.24 & 47.25 & 47.53 & 33.36 & 22.28 & 21.42 & 35.49 & 33.94 & 32.07 & \\
\hline 34.01 & 47.55 & 51.02 & 34.38 & 22.7 & 23.27 & 36.43 & 36.74 & 32.54 & \\
\hline
\end{tabular}

Tabela 9: Peso dos exemplares fêmeas

\begin{tabular}{|l|l|l|l|l|l|l|l|l|l|}
\hline 1.4320 & 1.0501 & 2.6734 & 0.8450 & 0.8400 & 0.3491 & 0.3041 & 1.3222 & 0.1810 & 1.0000 \\
\hline 1.9760 & 1.1651 & 1.9443 & 0.8450 & 0.8470 & 0.3616 & 0.6554 & 0.4503 & 0.6240 & 0.9000 \\
\hline 0.6557 & 1.6699 & 1.0486 & 0.9870 & 0.6159 & 0.4034 & 0.7548 & 0.7368 & 0.6430 & 1.1000 \\
\hline 0.1338 & 2.2340 & 1.0919 & 1.0540 & 1.2400 & 0.5026 & 0.8395 & 0.6613 & 1.0360 & \\
\hline 0.1821 & 3.3526 & 1.2176 & 0.6870 & 1.8660 & 0.8000 & 1.0468 & 0.7617 & 2.7344 & \\
\hline 0.3674 & 0.5385 & 1.6800 & 1.0840 & 2.4100 & 0.8470 & 0.9673 & 0.8178 & 0.3365 & \\
\hline 0.3954 & 1.9346 & 1.6128 & 0.9880 & 0.1543 & 0.7000 & 0.8686 & 0.6810 & 0.9000 & \\
\hline 0.4684 & 2.3606 & 1.5885 & 1.0850 & 0.1831 & 0.2140 & 1.1533 & 0.9909 & 1.0000 & \\
\hline 0.9600 & 2.0477 & 2.5702 & 0.5680 & 0.2093 & 1.0213 & 0.8841 & 1.1352 & 1.2000 & \\
\hline 0.6576 & 2.4792 & 2.9119 & 0.6105 & 0.3133 & 0.3019 & 1.2004 & 1.1352 & 1.0000 & \\
\hline
\end{tabular}

Tabela 10: Altura do corpo medida à frente da dorsal dos exemplares fêmeas

\begin{tabular}{|c|c|c|c|c|c|c|c|c|c|}
\hline 16.10 & 13.54 & 20.49 & 10.78 & 11.70 & 7.70 & 6.41 & 15.82 & 6.06 & 12.41 \\
\hline 18.10 & 13.70 & 16.93 & 10.73 & 14.86 & 8.87 & 10.11 & 9.67 & 10.35 & 13.64 \\
\hline 12.44 & 15.10 & 14.52 & 11.08 & 11.17 & 8.19 & 14.93 & 11.92 & 10.49 & 13.17 \\
\hline 5.75 & 16.54 & 15.45 & 11.85 & 15.65 & 9.01 & 14.87 & 11.23 & 12.53 & \\
\hline 6.00 & 20.88 & 15.70 & 12.69 & 19.51 & 11.75 & 16.35 & 11.40 & 21.13 & \\
\hline 8.76 & 10.85 & 13.93 & 10.56 & 23.24 & 11.21 & 13.22 & 12.19 & 8.69 & \\
\hline 8.99 & 18.92 & 17.79 & 12.22 & 5.69 & 11.82 & 13.91 & 11.10 & 12.77 & \\
\hline 9.21 & 19.53 & 17.69 & 11.01 & 6.33 & 5.22 & 13.35 & 13.24 & 12.85 & \\
\hline 10.79 & 19.52 & 19.54 & 13.25 & 7.23 & 6.20 & 13.23 & 12.03 & 12.83 & \\
\hline 11.56 & 20.00 & 20.70 & 18.04 & 8.06 & 6.66 & 14.53 & 13.31 & 12.59 & \\
\hline
\end{tabular}

\section{Referências}

[1] Barroso, L. C., Araújo B., Campos Filho, F. F, Carvalho, M.L., Maia, M.L. Cálculo Numérico (Com Aplicações). São Paulo: Ed. Harbra, 1987.

[2] Bassanezi, R. C.; Ferreira J. W. C. Equações Diferenciais com Aplicações. São Paulo: Ed. Harbra, 1988.

[3] Buckup, P.A. The Eastern Brazilian Shield. Historical Biogeography of Neotropical Freshwater

Fishes (J.S. Albert; R.E. Reis, Eds.). University of California Press, California, p. 203-210, 2011. 
[4] Cunha, M. C. C. Métodos Numéricos. $2^{\mathrm{a}}$ ed. Ed. da Unicamp, 2000.

[5] Delgado, J; Frensel. K; Crissaff, L. Geometria Analítica, Coleção Profmat. Rio de Janeiro: Ed. da SBM, 2013.

[6] Guerra, A. J. T., Bezerra, J. F. R., Jorge, M. do C. O., \& Fullen, M. A. "The Geomorphology Of Angra dos Reis and Paraty Municipalities, Southern Rio de Janeiro State". Revista Geonorte, v. $4, \mathrm{n}^{\mathrm{O}} 13$, p. 1-21, 2013.

[7] Iezzi, G; Hazzan, S. Fundamentos de Matemática Elementar 4. $2^{\mathrm{a}}$ ed. São Paulo: Atual Ed. Ltda., 1977.

[8] Kavalco, K. F., Brandão, K. de O., Pazza, R., Almeida-Toledo, L. F. de. (2009). "Astyanax Hastatus Myers, 1928 (Teleostei, Characidae): A New Species Complex Within The Genus Astyanax?" Genetics and Molecular Biology, v. 32, nº 3, p. 477-483. 2009.

[9] Mazzoni, R., Rezende, C. F. "Seasonal Diet Shift In a Tetragonopterinae (Osteichthyes, Characidae) From The Ubatiba River, RJ, Brazil". Braz. Journal Biology, v. 63, n⿳⺈ 1, p. 69-74, 2003.

[10] Pereira, W. R. L. S. "Alometrias Em Toda Parte". Revinter Revista Intertox de Toxicologia, Risco Ambiental e Sociedade, v. 6, $\mathrm{n}^{\mathrm{o}}$ 1, p. 78-101, Fev. 2013.

[11] Ruggiero M. A.; Lopes V. L. Cálculo Numérico. Aspectos Teóricos e Computacionais. ed. Makron Books, 1997.

[12] Souza-Lima, R.; Silva, J.C.M. da; Portugal, A. "Ictiofauna do rio Aldeia", São Gonçalo. In: Marcelo Guerra Santos. (Org.). Estudos Ambientais em Regiões Metropolitanas: São Gonçalo. $1^{\mathrm{a}}$ ed. Rio de Janeiro: Editora da Universidade do Estado do Rio de Janeiro, 2012, v. 1, p. 115134 .

[13] Spiegel, M. Estatística. Coleção Schaum. Rio de Janeiro: Ed. LTC, 1961.

June Cunha de Araujo Colégio Estadual Pandiá Calógeras

São Gonçalo, RJ $<$ junearaujo75@gmail.com>

Rosa García Márquez

Universidade do Estado do Rio de Janeiro <rosagmarquez@yahoo.com.br>

Jorge Corrêa de Araújo Universidade do Estado do Rio de Janeiro $<$ jcaraujo_55@yahoo.com.br>

Rosana Souza-Lima Universidade do Estado do Rio de Janeiro <rosanas1@yahoo.com.br>

Recebido: 28/06/2021

Publicado: 10/11/2021 